University of Nebraska - Lincoln

DigitalCommons@University of Nebraska - Lincoln

1979

\title{
Energy and Angular Distributions of Secondary Electrons from 5-100-keV-Proton Collisions with Hydrogen and Nitrogen Molecules
}

M. Eugene Rudd

University of Nebraska - Lincoln, erudd@unl.edu

Follow this and additional works at: https://digitalcommons.unl.edu/physicsrudd

Part of the Physics Commons

Rudd, M. Eugene, "Energy and Angular Distributions of Secondary Electrons from 5-100-keV-Proton Collisions with Hydrogen and Nitrogen Molecules" (1979). M. Eugene Rudd Publications. 67. https://digitalcommons.unl.edu/physicsrudd/67

This Article is brought to you for free and open access by the Research Papers in Physics and Astronomy at DigitalCommons@University of Nebraska - Lincoln. It has been accepted for inclusion in M. Eugene Rudd Publications by an authorized administrator of DigitalCommons@University of Nebraska - Lincoln. 


\section{Physical Review A 20, 787 - 796 (1979)}

\section{Energy and angular distributions of secondary electrons from 5-100-keV-proton collisions with hydrogen and nitrogen molecules}

M. E. Rudd

Behlen Laboratory of Physics, University of Nebraska, Lincoln, Nebraska 68588

Received 9 March 1979

Cross sections for the ejection of electrons from hydrogen and nitrogen by protons have been measured as a function of the energy and angle of ejection of the electrons at incident proton energies of 5-70 keV and $100 \mathrm{keV}$ for hydrogen. The range of angles measured was $10-160^{\circ}$ and the electron energy range was $1.5-300 \mathrm{eV}$. The doubly differential cross sections were also integrated over angle, over electron energy, or over both to obtain singly differential and total cross sections for electron production. Average electron energies were also calculated from the data. The angular distributions of electrons are peaked in the forward direction but become more isotropic as the proton energy decreases. Nitrogen yields a more isotropic distribution than hydrogen. In this range of proton energies the cross sections integrated over angle are found to fall off approximately exponentially with electron energy, and a simple empirical equation has been found that describes the singly differential and total cross sections within a factor of 2 for several targets. A theoretical interpretation of this result in terms of the molecular promotion model is given in which Meyerhof's method of calculating cross sections for $K$-shell excitation is applied for the first time to the ionization of outer shells of atoms.

(C)1979 The American Physical Society

URL: http://link.aps.org/doi/10.1103/PhysRevA.20.787

DOI: 10.1103/PhysRevA.20.787 


\title{
Energy and angular distributions of secondary electrons from 5-100-keV-proton collisions with hydrogen and nitrogen molecules
}

\author{
M. E. Rudd \\ Behlen Laboratory of Physics, University of Nebraska, Lincoln, Nebraska 68588
}

(Received 9 March 1979)

\begin{abstract}
Cross sections for the ejection of electrons from hydrogen and nitrogen by protons have been measured as a function of the energy and angle of ejection of the electrons at incident proton energies of 5-70 keV and $100 \mathrm{keV}$ for hydrogen. The range of angles measured was $10-160^{\circ}$ and the electron energy range was 1.5-300 eV. The doubly differential cross sections were also integrated over angle, over electron energy, or over both to obtain singly differential and total cross sections for electron production. Average electron energies were also calculated from the data. The angular distributions of electrons are peaked in the forward direction but become more isotropic as the proton energy decreases. Nitrogen yields a more isotropic distribution than hydrogen. In this range of proton energies the cross sections integrated over angle are found to fall off approximately exponentially with electron energy, and a simple empirical equation has been found that describes the singly differential and total cross sections within a factor of 2 for several targets. A theoretical interpretation of this result in terms of the molecular promotion model is given in which Meyerhof's method of calculating cross sections for $K$-shell excitation is applied for the first time to the ionization of outer shells of atoms.
\end{abstract}

\section{INTRODUCTION}

The study of the angular and energy distributions of electrons ejected from target atoms and molecules by protons has provided data of use in a wide range of applied areas such as upper atmospheric studies, ion bombardment of surfaces, radiation damage of biological materials, and tracks in nuclear emulsions. While earlier measurements of this kind ${ }^{1}$ were confined to proton energies from $50 \mathrm{keV}$ to $5 \mathrm{MeV}$, two recent papers, one on helium ${ }^{2}$ and the other on argon, ${ }^{3}$ have extended the energy range downward to $5 \mathrm{keV}$, a range of even greater applied interest. While these two low-energy studies were for protons on monatomic gases, the present work studies two diatomic molecules, hydrogen and nitrogen, as targets. The data on nitrogen should be of especial interest in auroral work since most of the protons entering the atmosphere are in the present energy range.

A relatively simple empirical equation describing the energy distribution of electrons integrated over all angles is presented in this paper; this equation provides a fit to the cross sections for all gases for which data are available and at all proton energies below the maximum in the totalcross-section curve. This equation is easily integrated in various ways to provide, e.g., estimates of the numbers of secondary electrons in any given energy range, average energies of ejection, and total electron production cross sections.

In addition to its applied interest, data of this type have proved valuable in elucidating the mechanisms for secondary electron production. Doubly differential cross sections provide a much more rigorous test of proposed theoretical descriptions than total cross sections. For example, studies of this kind led to the discovery of the mechanism of charge transfer to the continuum ${ }^{4}$ and also formed part of the basis for the development of the molecular promotion model by Fano and Lichten. ${ }^{5}$ The present paper applies the promotion model to yield a quantitative description of the energy distribution of electrons ejected in low-energy ion-atom or ion-molecule collisions. This method may prove useful in a region where highenergy techniques, such as the Born approximation, are of restricted value.

\section{EXPERIMENTAL METHOD}

Since the apparatus and techniques used in this investigation were the same as for the earlier helium work, ${ }^{2}$ only a brief description will be given here. Protons from an rf ion source were accelerated, analyzed magnetically, and finely collimated before entering the collision chamber, where they were caught by a deep, unbiased Faraday cup. Target-gas pressure was measured by a capacitance manometer. Electrons ejected from the static gas in the path of the beam passed through a pair of defining slits into a $127^{\circ}$ electrostatic analyzer. The slit system and analyzer could be placed at any of nine fixed angles from 10 to $160^{\circ}$ from the proton beam. The angular acceptance was $\pm 1.5^{\circ}$ and the energy resolution of the analyzer was $4.4 \%$. No preacceleration was used. The Earth's magnetic field was annulled by three Helmholtz coil pairs to less than $5 \mathrm{mG}$. The de- 
tector was a 17-stage electron multiplier with its first dynode biased at $82 \mathrm{~V}$ to produce a nearly constant detection efficiency for all electron energies from 1.5 to $300 \mathrm{eV}$. For the hydrogen data the efficiency was measured as previously described and found to be $0.62 \pm .05$. Some of the data were measured at a different efficiency and then normalized to data retaken with the known efficiency. In the case of nitrogen, the efficiencies were not known as well, so the data were normalized separately for each angle to the $50-\mathrm{keV}$ data of Crooks and Rudd. ${ }^{6}$

At the higher proton energies the uncertainty in the cross sections at most angles and electron energies was $20 \%$. At the lower proton energies smaller beam currents and cross sections required longer integration times and entailed greater uncertainties. In addition, contamination of the beam with neutrals was a greater problem. As is usual in this work, electrons below about $10 \mathrm{eV}$ are difficult to control because of stray electric and magnetic fields. As the proton energy was lowered, these low-energy electrons formed a larger fraction of the total production so that integration over electron energy introduced larger uncertainties. While no single number describes the uncertainties at all values of the various parameters, some data at the lowest proton energy may have uncertainties as large as a factor of 2 .

\section{EXPERIMENTAL RESULTS}

Doubly differential cross sections were measured at all combinations of target gas, ejected-electron energy, proton energy, and angle. The proton energies were $5,7,10,15,20,30,50$, and $70 \mathrm{keV}$ for both gases and $100 \mathrm{keV}$ for hydrogen. Because several parameters are involved, there are many ways in which the data can be displayed. Figure 1 shows the energy distributions of the electrons from hydrogen ejected at various angles by $20-\mathrm{keV}$ protons. No theoretical calculations are known that have been made using realistic molecular wave functions, but a comparison is made for the two angles 10 and $110^{\circ}$ with Born-approximation calculations using hydrogenic wave functions scaled to the ionization potential of molecular hydrogen, $15.422 \mathrm{eV}$. Agreement at the higher electron energies is quite good, but even accounting for a possibly large experimental error at low energies, the agreement there is poor. At small angles the cross section is underestimated by the Born approximation, while at other angles it is overestimated. The decrease in the cross sections with electron energy is seen to be approximately exponential. As shown later, this exponential behavior is followed even more closely by cross sec-

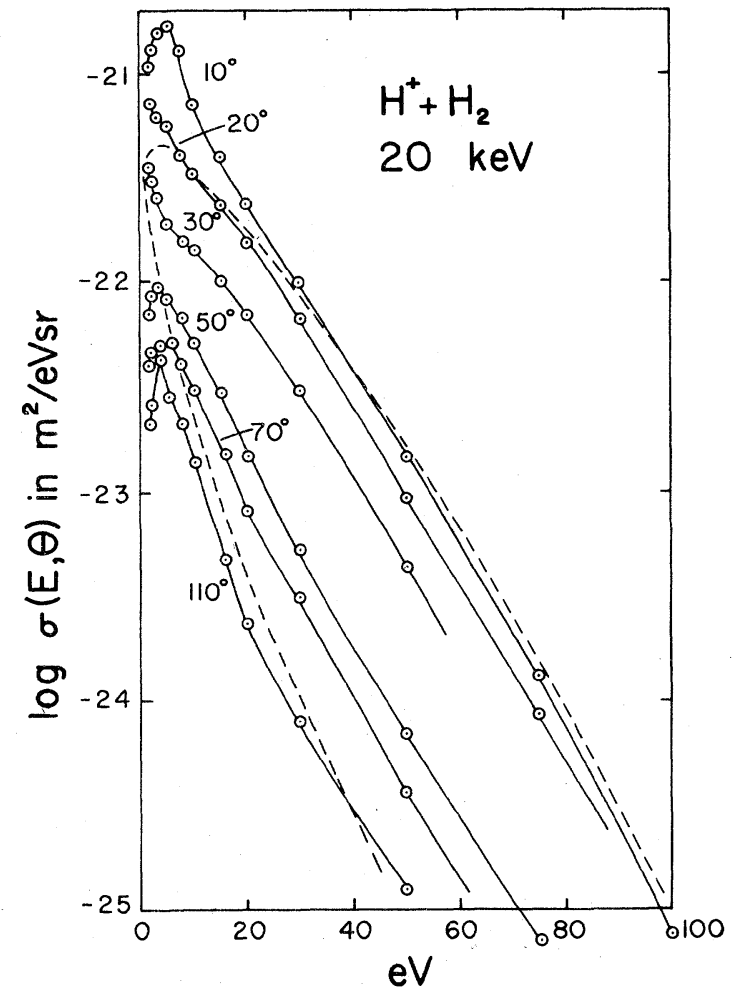

FIG. 1. Doubly differential cross sections for electrons ejected at various angles as a function of ejection energy for 20-keV-proton impact on hydrogen gas. Points and solid lines are the present data; dashed lines are Born-approximation calculations at 10 and $110^{\circ}$.

tions which have been integrated over all angles of ejection.

The angular distribution of the cross sections is peaked in the forward direction but is roughly constant at large angles with evidence of some structure. Figure 2 shows the data for nitrogen at 7 $\mathrm{keV}$. In general, the lower the proton energy and the lower the ejected-electron energy, the more isotropic the distributions become. Electrons from nitrogen are distributed more isotropically than those from hydrogen.

Figure 3 illustrates the dependence of the $90^{\circ}$ cross sections for nitrogen on proton impact energy for various electron energies. Data from other investigations have been added to make a more complete graph. As with data on helium presented earlier, ${ }^{2}$ the general trend shows a falling off at both high and low proton energies with a maximum at an intermediate energy. As with the helium curves, there is an unexplained dip, less pronounced than for helium, not far from the point where the proton velocity equals that of the ejected electron.

Figures 4 and 5 show more clearly the compari- 


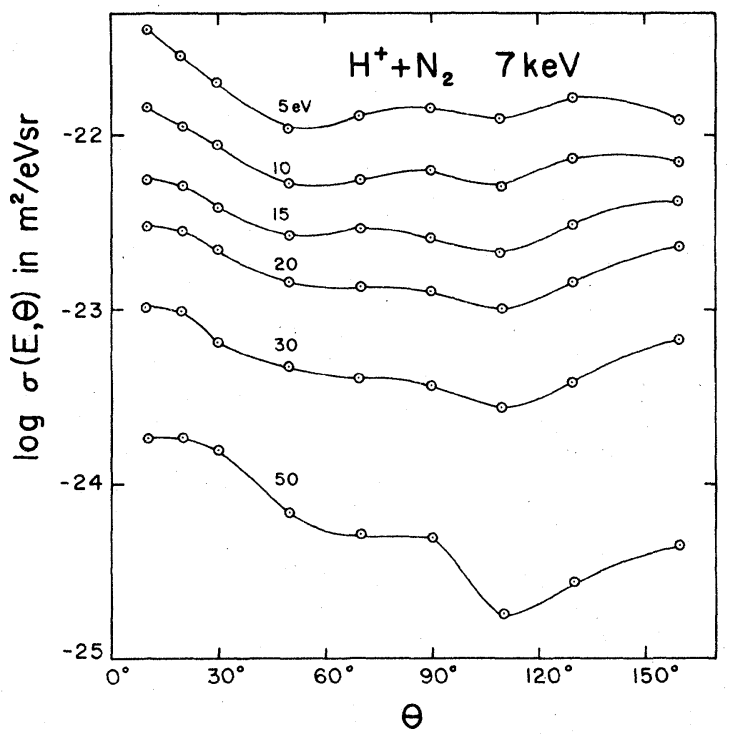

FIG. 2. Angular distributions of electrons ejected at various energies by $7-\mathrm{keV}$-proton impact on nitrogen gas.

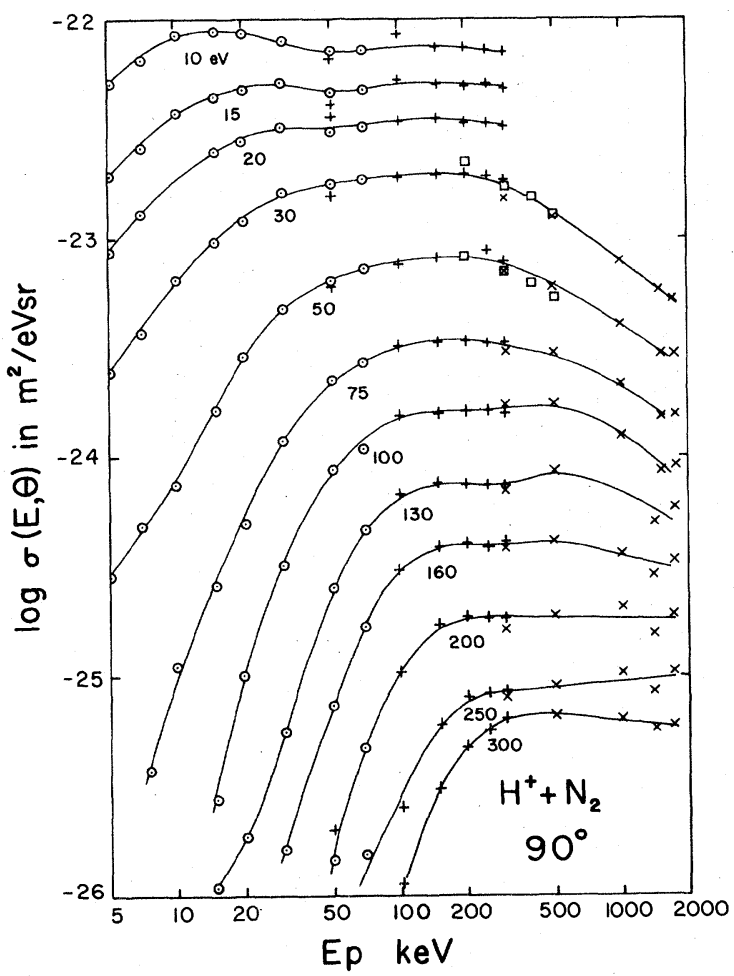

FIG. 3. Doubly differential cross sections for electron ejection at $90^{\circ}$ from nitrogen for various electron energies as a function of proton energy: $O$, present data; + , data of Crooks and Rudd (Ref. 6); $\times$, data of Toburen (Ref. 7); 口, data of Stolterfoht (Ref. 8). son of the angular distributions between hydrogen and nitrogen targets. In these graphs are plotted the singly differential cross sections which have been integrated over electron energy at each angle. Also shown are comparisons to earlier work in the regions of overlap. At the lower energies, especially in the case of hydrogen, structures appear in the angular distributions which persist even after integration over electron energy. Although it is likely that these features are real, one must bear in mind that for the apparatus used here it is necessary to let the collision chamber, analyzer, and detector return to atmospheric pressure to change angles. Therefore data at adjacent angles may have been taken at quite different times with possible changes in detector efficiency affecting the normalization. In addition, the angular mesh used is not fine enough to allow a definitive statement about this structure. However, similar structures were seen in argon by Criswell and Toburen, ${ }^{3}$ who used finer angular steps.

When the double differential cross sections are integrated instead over all angles, we get the results shown in Figs. 6 and .7. At about $50 \mathrm{keV}$,

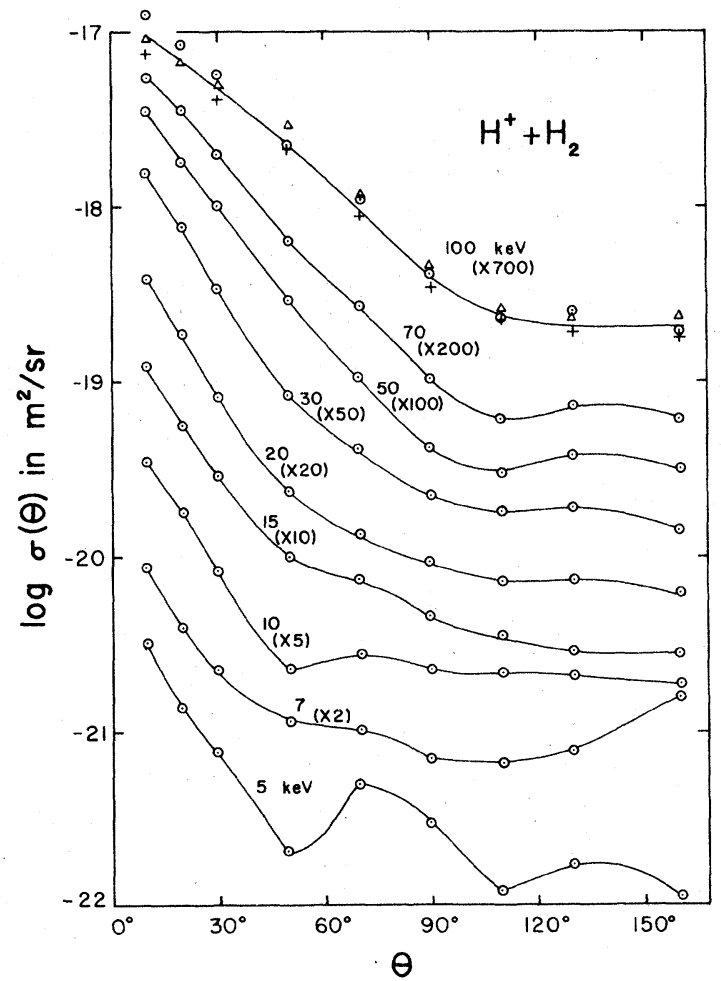

FIG. 4. Angular distributions of singly differential cross sections for ejection of electrons from hydrogen by protons of various energies: 0 , present data; $\Delta$, data of Rudd et al. (Ref. 9); +, data of Rudd and Jorgensen (Ref, 10). 


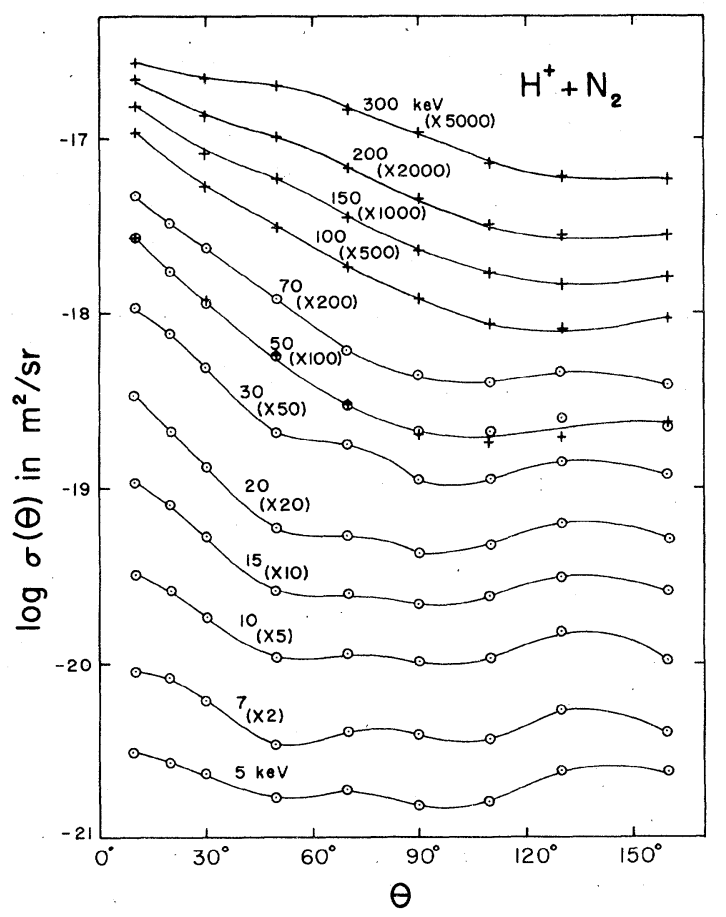

FIG. 5. Angular distributions of singly differential cross sections for ejection of electrons from nitrogen by protons of various energies: 0 , present data; + , Crooks and Rudd (Ref.6).

especially in hydrogen, one sees the beginning of the binary-encounter peak which becomes dominant at $\mathrm{MeV}$ energies. At lower energies, however, it becomes less important and is not visible in the integrated cross sections below $50 \mathrm{keV}$. As noted before, ${ }^{2}$ the forward peak from the mechanism of charge transfer to the continuum also becomes negligibly small at low energies. With these two features absent, the energy distributions at low proton energy are very regular and not far from straight lines on a semilogarithmic plot. This regularity suggests that a simple equation can be found to fit the curves, a possibility which is pursued further in Sec. IV.

Total cross sections for electron production may be obtained by integrating the doubly differential cross sections over both angle and electron energy. Values obtained in this way are given in Table I for both target gases. Also given are average energies of electron ejection as determined from the experimental data.

\section{EMPIRICAL FIT TO ENERGY DISTRIBUTIONS}

As noted, below $50 \mathrm{keV}$ the dependence of the singly differential cross sections on electron energy appears from Figs. 6 and 7 to be nearly exponential. To find an equation which fits these curves and similar ones for other targets the slopes were first measured to determine the dependence on proton energy $E_{p}$. The slope was found to follow an $E_{p}^{-1 / 2}$ dependence. Then, using data previously published for helium ${ }^{2}$ and argon $^{3}$ at low energies and neon and oxygen at $50 \mathrm{keV},{ }^{6}$ it was possible to see an $I^{-1 / 2}$ dependence of the slope, where $I$ is the ionization potential or binding energy of the electrons in the atom or molecule. The shapes of the curves can, in fact, be fit quite well by the expression $\exp \left[-\alpha E /(I T)^{1 / 2}\right]$, where $E$ is the ejected-electron energy, $T=E_{p} / 1836$ $=\frac{1}{2} m_{e} v_{p}^{2}$, and $\alpha$ is a dimensionless constant not much different from unity. The best fits are obtained by choosing $\alpha=1.28$ for $\mathrm{H}_{2}$ and $\mathrm{He}$ and $\alpha$ $=0.91$ for $\mathrm{N}_{2}, \mathrm{O}_{2}, \mathrm{Ne}$, and Ar.

A complete equation for the singly differential cross sections would include a coefficient for the exponential expression that depends on $T$ and $I$. An equation that has been found to work well contains two "semiadjustable" dimensionless parameters, $\alpha$ and $\beta$. They are semiadjustable in the sense that all of the gases tested fall into one or the other of two categories, each with its set of values of these parameters. When dealing with target atoms or molecules with more than one subshell, reasonably good results can be obtained by using the same expression for all the subshells, each with its own characteristic binding energy, and then adding the resulting partial cross sections to obtain the cross section for the entire atom or molecule. The equation is

$\sigma(E)=5 \pi a_{0}^{2} \alpha^{3} \sum_{i} \frac{N_{i} I_{H}^{2}}{I_{i}^{3}} \frac{\left(T / I_{i}\right)^{\beta-1 / 2}}{4+\left(T / I_{i}\right)^{2 B}} \exp \left(-\frac{\alpha E}{(I T)^{1 / 2}}\right)$,

where for $\mathrm{H}_{2}$ and He targets $\alpha=1.28$ and $\beta=1.0$, while for $\mathrm{N}_{2}, \mathrm{O}_{2}, \mathrm{Ne}$, and $\operatorname{Ar} \alpha=0.91$ and $\beta=0.75$. $N_{i}$ is the number of electrons in the target with binding energy $I_{i}, I_{H}=13.6 \mathrm{eV}$, and $a_{0}$ is the radius of the first Bohr orbit.

Figure 8 shows the extent of the fit of Eq. (1) for various energies of protons on hydrogen gas. The experimental cross sections are divided by the values calculated from Eq. (1). Even though the cross sections themselves vary by five or six orders of magnitude over the range of electron energies measured, the division by the exponential expression yields results which vary by less than a factor of 2 everywhere below $50 \mathrm{keV}$. This agreement with experiment is better than obtained by calculations from the Born approximation (even with realistic wave functions) or from the binaryencounter approximation and far better than the results of Rutherford's equation. Above $50 \mathrm{keV}$, the cross sections depart significantly from the 


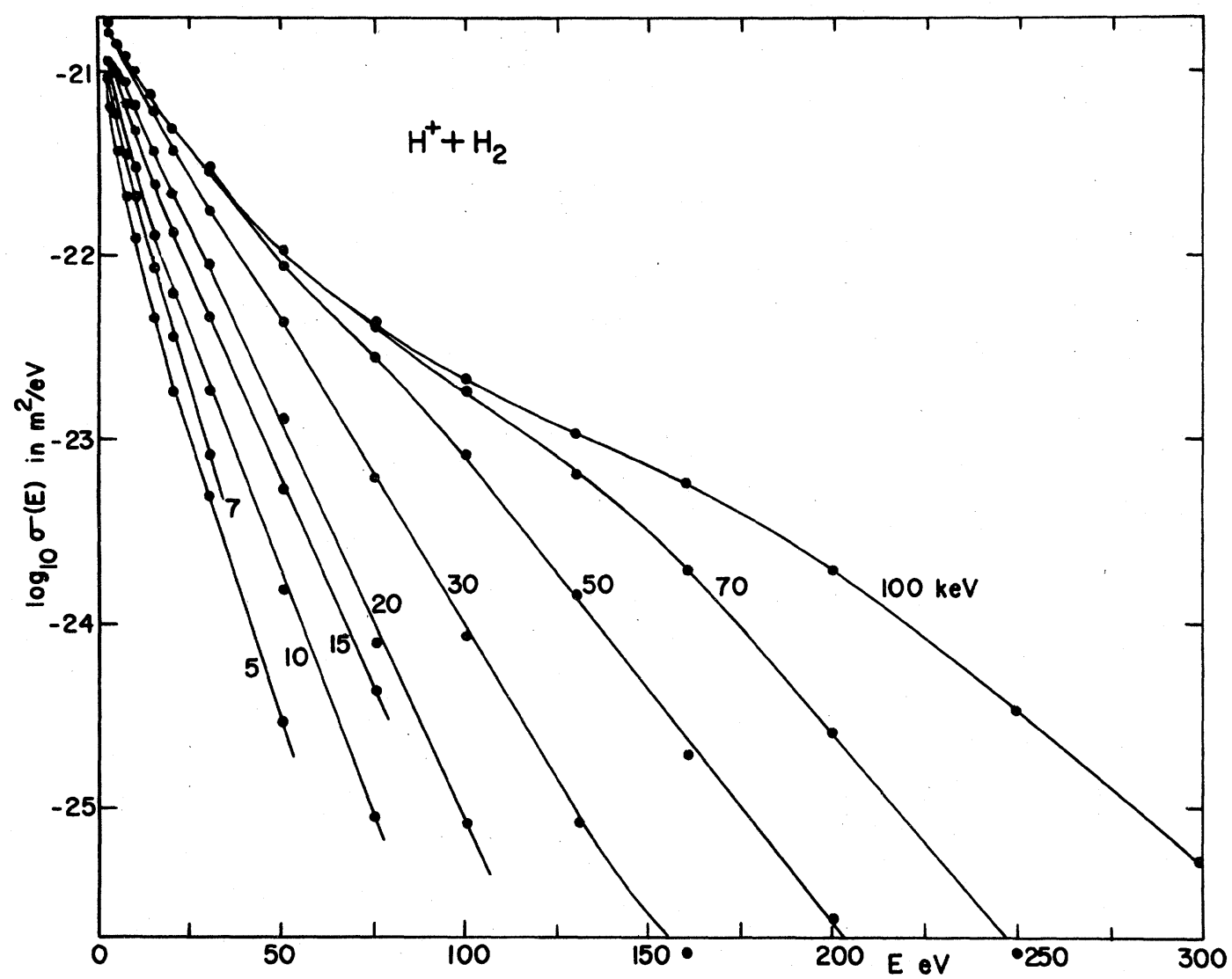

FIG. 6. Energy distributions of electrons integrated over all angles for various energies of proton impact on hydrogen.

results of Eq. (1), especially near $E=4 T$, where the binary-encounter peak now becomes important. In Fig. 9 the same ratio is plotted for four different target gases at $20 \mathrm{keV}$. The poorest fit is that of helium, but it is still within a factor of 2 at nearly all energies.

To test the application of Eq. (1) to inner shells, Figs. 10 and 11 were drawn showing the comparison of experimental data and calculations for the various shells of argon and nitrogen. Appropriate binding energies for the molecular-nitrogen shells were obtained from Siegbahn. ${ }^{11}$ The loosely bound outer shells contribute most to the cross sections at low energies but fall off rapidly. At higher energies the inner shells with smaller slopes begin to contribute significantly and become dominant at a high enough energy. Note in the case of argon that above $300 \mathrm{eV}$ the slope of the experimental

TABLE I. Total electron production cross sections and average ejected-electron energies.

\begin{tabular}{rlccc}
\hline $\begin{array}{c}\text { Proton } \\
\text { energy } \\
(\mathrm{keV})\end{array}$ & \multicolumn{2}{c}{ Total cross sections } & \multicolumn{2}{c}{$\begin{array}{c}\text { Average electron energies } \\
(\mathrm{eV})\end{array}$} \\
\hline & Hydrogen & Nitrogen & Hydrogen & Nitrogen \\
\hline 5 & $5.62 \times 10^{-21}$ & $2.46 \times 10^{-20}$ & 5.46 & 5.42 \\
7 & 7.75 & 2.79 & 6.47 & 6.67 \\
10 & 9.16 & 3.22 & 6.91 & 7.74 \\
15 & 13.1 & 3.78 & 8.81 & 9.99 \\
20 & 16.9 & 4.22 & 10.2 & 11.9 \\
30 & 24.9 & 4.82 & 12.7 & 15.2 \\
50 & 32.0 & 5.33 & 16.0 & 20.4 \\
70 & 31.2 & 5.33 & 19.4 & 24.4 \\
100 & 26.5 & & 22.9 & \\
\hline
\end{tabular}




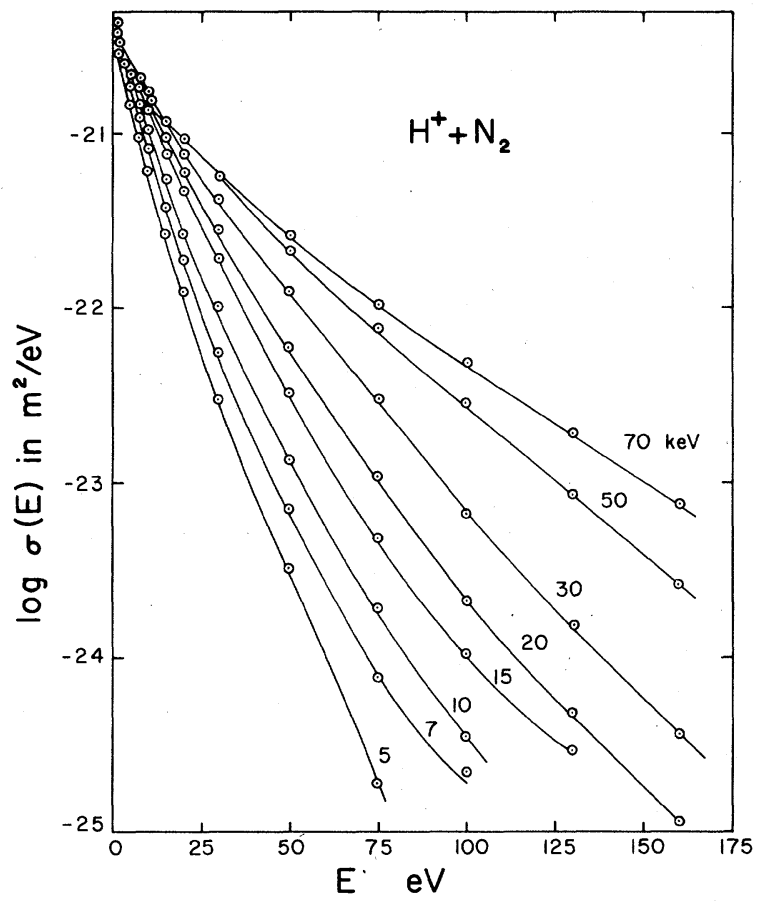

FIG. 7. Energy distributions of electrons integrated over all angles for various energies of proton impact on nitrogen.

curve matches that of the $2 p$ subshell although the calculations are nearly a factor of 2 too low in value. If the $2 p$ cross sections, calculated from Eq. (1), are integrated over all electron energies, the result should equal the Auger ionization cross section represented by the area under the peak between 150 and $210 \mathrm{eV}$. The results of the integration are too small by a factor of 2 also, which indicates that, while the coefficient of the exponential factor in Eq. (1) may need to be modified for inner-shell ionization, the basic functional form probably is accurate. It may be that Eq. (1) could be improved, e.g., by choosing a different value of the parameter $\beta$ for inner shells, but this possibility has not been explored.

Equation (1) can be integrated over all electron energies to obtain the total cross section for electron production. The result is

$$
\sigma_{e}=5 \pi\left(a_{0} \alpha\right)^{2} \sum_{i} \frac{N_{i} I_{H}^{2}}{I_{i}^{2}} \frac{(T / I)^{\beta}}{4+(T / I)^{2 \beta}} .
$$

This functional form of $T$ is similar to one given by Green and $\mathrm{McNeal}^{12}$ to fit ionization cross sections. However, they used a single term with additional parameters rather than a summation as here. The results of this calculation are shown in Fig. 12 for three gases along with experimental data. The agreement is reasonably good even at the higher proton energies, where the exponential

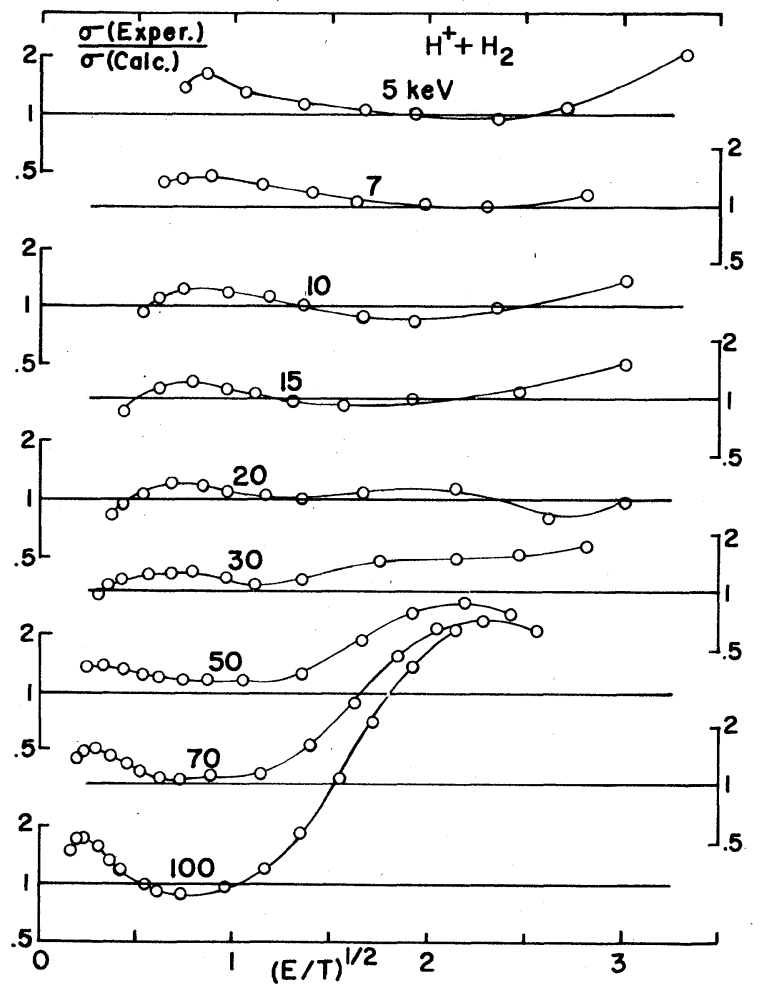

FIG. 8. Ratio of experimental singly differential electron-ejection cross sections integrated over all angles to values calculated from Eq. (1) at various energies of proton impact on hydrogen.

dependence on $E$ of Eq. (1) fails.

Though purely empirical, Eqs. (1) and (2) should be useful to those who wish to estimate electronejection cross sections by low-energy protons and to calculate various other quantities related to secondary electron production. If the target atom contains just one subshell or if the sum in Eq. (1) is dominated by a single term, then a particularly simple calculation can be made for some quantities. For example, the average electron energy is then

$$
\bar{E}=\int_{0}^{\infty} E \sigma(E) d E / \int_{0}^{\infty} \sigma(E) d E=\frac{(I T)^{1 / 2}}{\alpha} .
$$

The fraction of electrons ejected with energies greater than the ionization potential (and thus able to cause further ionization) is

$$
f=\int_{I}^{\infty} \sigma(E) d E / \int_{0}^{\infty} \sigma(E) d E=\exp \left[-\alpha(I / T)^{1 / 2}\right] .
$$

Calculations from Eq. (3) are plotted in Fig. 13 for hydrogen along with the experimental values of the average electron energy. The agreement is very good at low energies but large deviations appear at higher energies, where Eq. (1) is no 


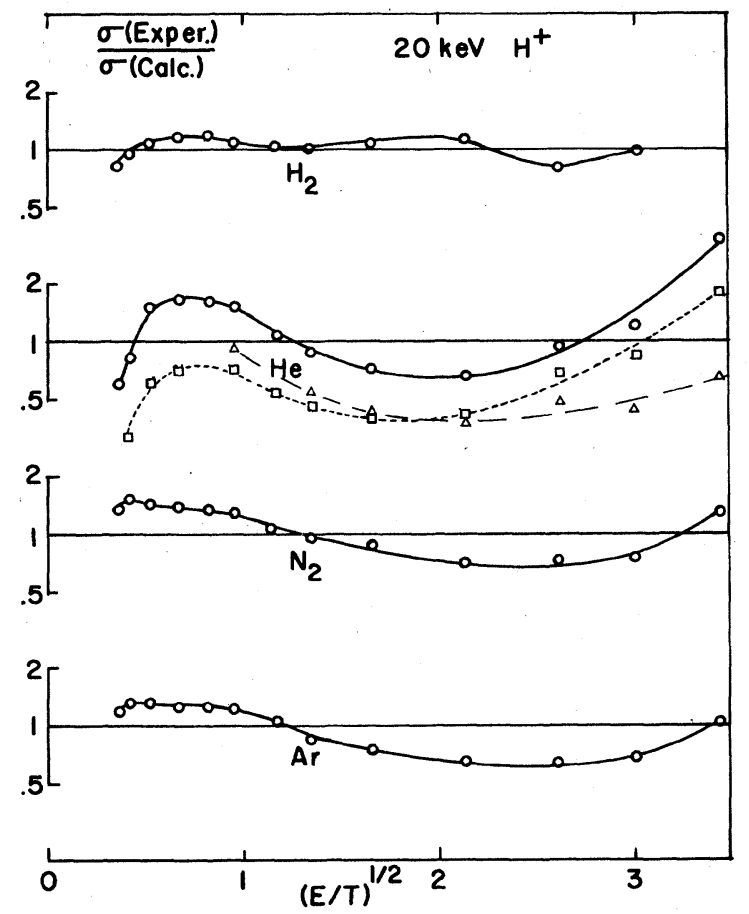

FIG. 9. Ratio of experimental singly differential cross sections integrated over all angles to calculated values at $20 \mathrm{keV}$ for four target gases: $O$, calculations from $\mathrm{Eq}$. (1); $\Delta$, Born Hartree-Fock calculations (Ref. 2); ㅁ, Born hydrogenic calculations scaled to helium.

longer valid.

An advantage of having an approximate mathematical form for the cross section curves is that by dividing by the empirical expression, the resulting curves are leveled out so that one can study small variations which are normally hidden in the large slope in the energy dependence. Referring to Fig. 8, one notes, e.g., that there is a small maximum at low electron energies which appears consistently at $E \simeq 0.6 T$ from 5 to $30 \mathrm{keV}$ but then disappears abruptly at $50 \mathrm{keV}$. This may be an indication that different mechanisms of electron production are dominant at proton energies above and below the maximum in the total-cross-section curve. A similar effect occurs for helium, but the transition is at a higher proton energy since the maximum in the total cross section appears at a higher energy.

\section{THEORETICAL JUSTIFICATION}

It is possible to derive the exponential dependence of Eq. (1) from the molecular-orbital promotion model. ${ }^{5}$ This derivation utilizes a result derived by Meyerhof ${ }^{18}$ for $K$-shell transitions and applied by Meyerhof and Taulbjerg ${ }^{19}$ to inner-shell

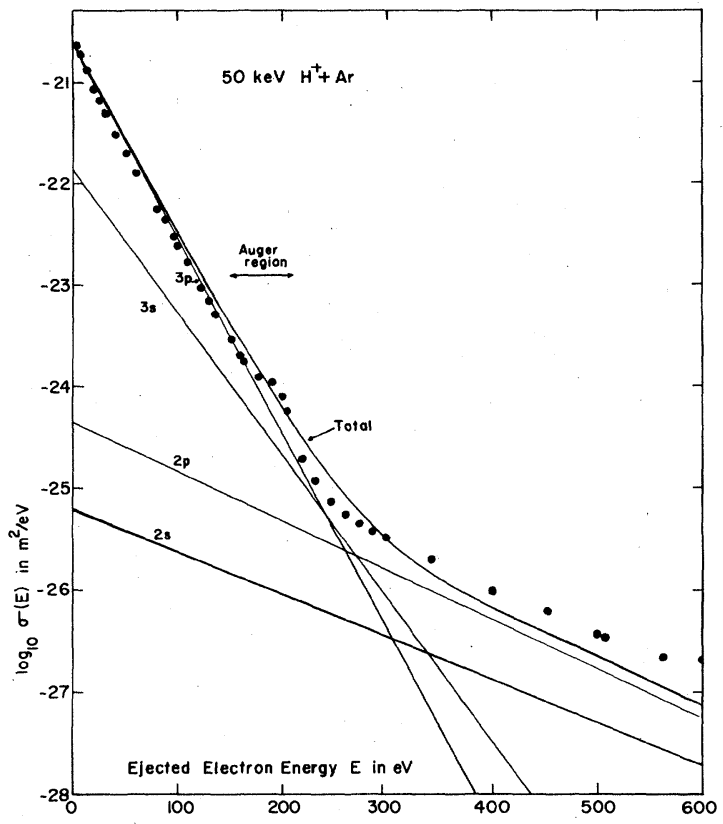

FIG. 10. Comparison of experimental and calculated cross sections for electrons ejected from argon by $50-$ $\mathrm{keV}$ protons: $\bullet$, data of Crooks and Rudd (Ref. 6); solid lines, calculations from Eq. (1) for separate subshells and the total for all shells.

ionization in symmetric collisions. We now propose to apply the model to outer-shell ionization by protons.

Consider the correlation diagram shown schematically in Fig. 14. As the proton approaches the target $X$ a charge transfer transition can take place with a probability $P_{1}$ involving the energy difference $\Delta E_{1}$. Then near the distance of closest approach a rotational coupling results in the promotion of the system to an excited state of either the hydrogen atom or the target $X$. In either case, the state has an energy $\Delta E_{2}$ only a few eV below the ionization continuum. Finally, a transition with an energy transfer of $\Delta E_{2}+E$ and a probability $P_{2}$ yields a free-electron of energy $E$. The cross section for the entire process can be written

$$
\sigma(E) \propto P_{1} \sigma_{\text {rot }} P_{2},
$$

where $\sigma_{\text {rot }}$ is the cross section for the rotational coupling transition. According to Taulberg, ${ }^{20}$ $\sigma_{\text {rot }}$ has a $v_{p}^{2 / 3}$ dependence on projectile velocity for nearly symmetric collisions. However, for these highly unsymmetric collisions this may not be accurate. Meyerhof ${ }^{18}$ has given an expression for the transition probabilities derived from Demkov's treatment ${ }^{21}$ of charge transfer. Using Meyerhof's result, we find $P_{1}=1 /\left(1+\exp 2 x_{1}\right)$ and $P_{2}$ $=1 /\left(1+\exp 2 x_{2}\right)$, where $x_{1}=\Delta E_{1} l_{1} / 2 \hbar v_{p}, x_{2}$ $=\left(E+\Delta E_{2}\right) l_{2} / 2 \hbar v_{p}$, and $v_{p}$ is the velocity of the 


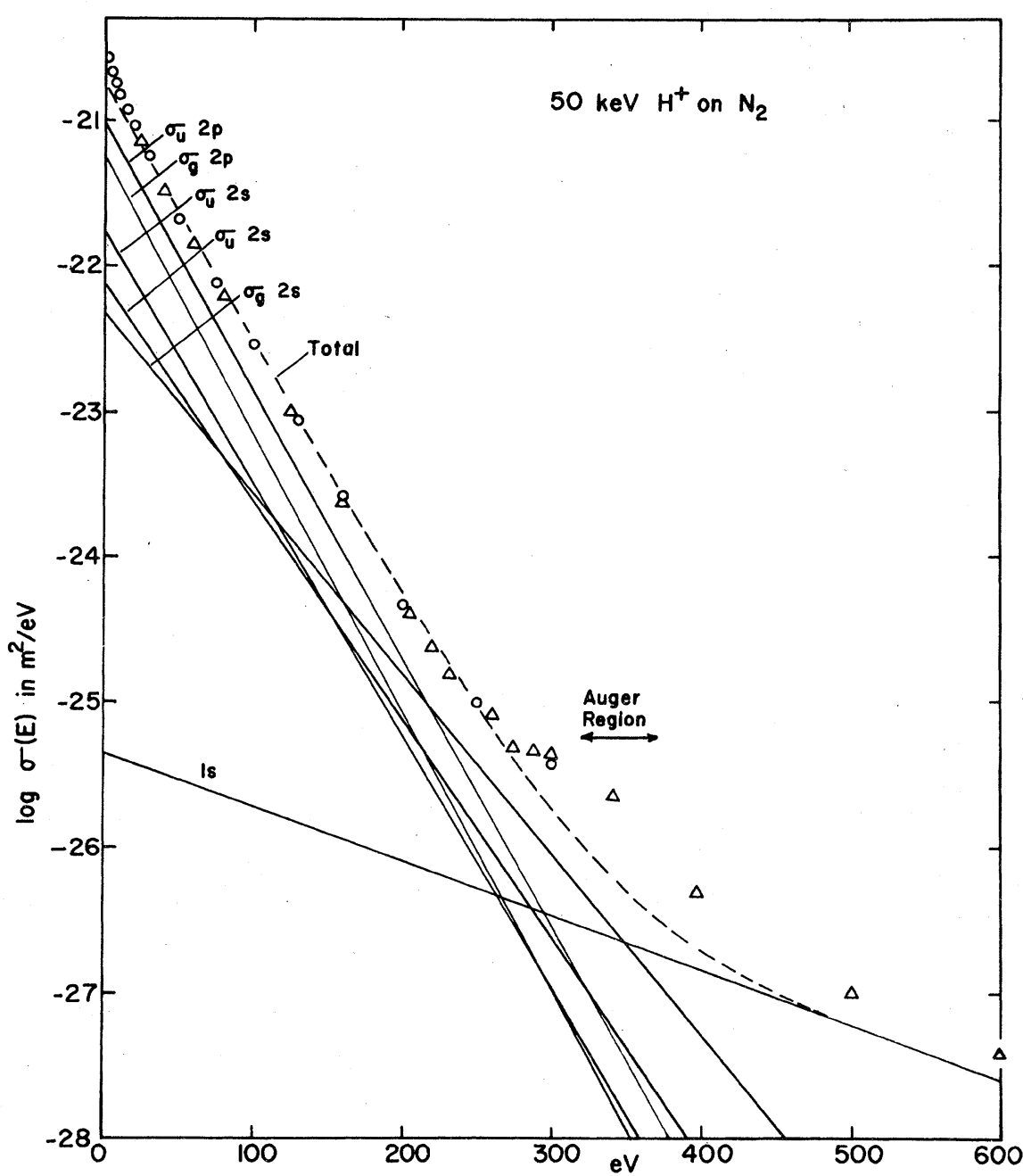

FIG. 11. Comparison of experimental and calculated cross sections for electrons ejected from nitrogen by $50-\mathrm{keV}$ protons: 0 , present results; $\Delta$, data of Crooks and Rudd (Ref. $6)$; solid lines, calculations from Eq. (1) for separate subshells; dotted line, sum of calculations for all subshells.

proton. The characteristic lengths $l_{1}$ and $l_{2}$ are not specified but may be taken as approximately equal to the diameter of the atomic shell. We set $l_{1}=l_{2}=2 \alpha a_{0}\left(I_{H} / I\right)^{1 / 2}$, where $\alpha$ is a dimensionless length parameter near unity. This parameter turns out to be identical to the constant $\alpha$ in the empirical equation given in Sec. IV. If $v_{0}$ is the velocity associated with the first Bohr orbit, then $v_{p}=v_{0}\left(T / I_{H}\right)^{1 / 2}$. Then, since $I_{H}=\hbar v_{0} / 2 a_{0}$, we may write

$$
\exp 2 x_{2}=\exp \alpha\left(E+\Delta E_{2}\right) /(I T)^{1 / 2} .
$$

If $T$ is small and $E$ is not too small we can make the approximation

$$
\begin{aligned}
\left(1+\exp 2 x_{2}\right)^{-1} & \simeq \exp -2 x_{2} \\
& =\exp \left(-\frac{\alpha \Delta E_{2}}{(I T)^{1 / 2}}\right) \exp \left(-\frac{\alpha E}{(I T)^{1 / 2}}\right),
\end{aligned}
$$

which is the same exponential dependence on ejected-electron energy as found empirically in
Sec. IV. Since $\alpha$ is identified here as a parameter associated with the size of the atomic shells, it is appropriate that it appears in Eq. (2) multiplying the factor $a_{0}$.

While the Meyerhof equation assumes transitions between bound states, the extension to continuum states involves only technical problems and has already been carried out. ${ }^{22}$ Because of the energy differences, it appears that the regions of interest for the three transitions are spatially separated, thus justifying the use of Meyerhof's equation in Eq. (5).

Going back to Eq. (5) with Meyerhof's expression inserted, we can integ rate over all electron energies without making the above approximation and get an expression for the total electron production cross sections. If we set $\alpha=1$, the result is

$$
\begin{aligned}
\sigma_{e} \propto & (T / I)^{5 / 6}\left(1+\exp \Delta E_{1} / T^{1 / 2} I^{1 / 2}\right)^{-1} \\
& \times \ln \left(1+\exp \Delta E_{2} / T^{1 / 2} I^{1 / 2}\right)
\end{aligned}
$$




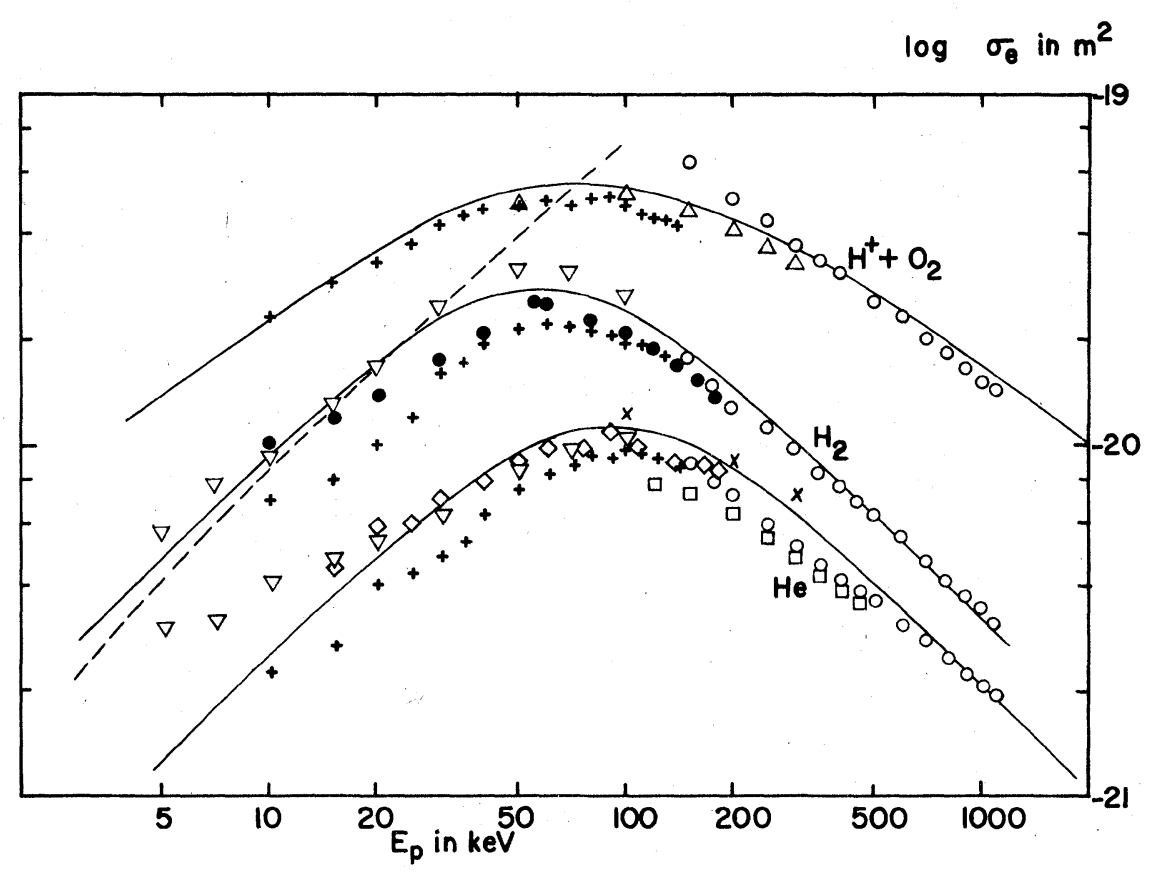

FIG. 12. Total cross sections for electron ejection from oxygen, hydrogen, and helium target gases by protons as a function of proton energy: $\nabla$, present data for hydrogen and data of Rudd and Madison (Ref. 2) for helium; $\bigcirc$, data of Hooper and co-workers (Ref. 13); +, data of DeH eer et al. (Ref.14); $\triangle$, data of Crooks and Rudd (Ref.6); $\times$, data of Rudd et al. (Ref. 9); $\diamond$, data of Solov'ev et al. (Ref. 15); $\square$, data of Gilbody and Lee (Ref. 16); $\bullet$, data of Afrosimov et al. (Ref. 17); solid lines, calculations using Eq. (2); dashed line, calculations for hydrogen using Eq. (3) with $\Delta E_{1}=1.8 \mathrm{eV}$, $\Delta E_{2}=3 \mathrm{eV}$, and $I=15.422$, normalized at $20 \mathrm{keV}$ to present data.

For hydrogen, $\Delta E_{1}=15.4-13.6 \mathrm{eV}=1.8 \mathrm{eV}$ and $\Delta E_{2}$ is approximately $3 \mathrm{eV}$. The resulting cross section is not sensitive to the values of $\Delta E_{1}$ and $\Delta E_{2}$ provided they are small. Calculations using Eq. (6) for hydrogen, normalized to the experimental data at $20 \mathrm{keV}$, are plotted as the dashed line in Fig. 12. The dependence on proton energy is dominated by the $T^{5 / 6}$ dependence from $\sigma_{\text {rot }}$ and

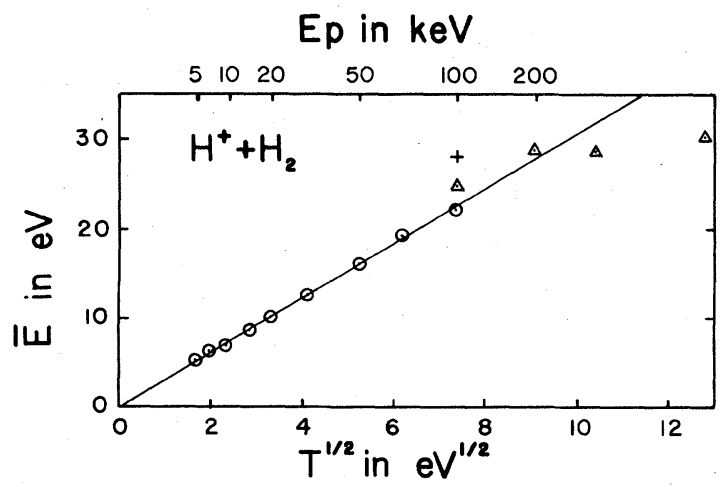

FIG. 13. Average energy of electrons ejected from hydrogen as a function of proton energy: 0 , present data; $\Delta$, data of Rudd et al. (Ref. 9) +, data of Rudd and Jorgensen (Ref. 10); solid line, calculation from Eq. (3). so is not much different from the first-power dependence on $T$ for hydrogen and helium or the $T^{0.75}$ dependence for other targets given by Eq. (2) at low energies.

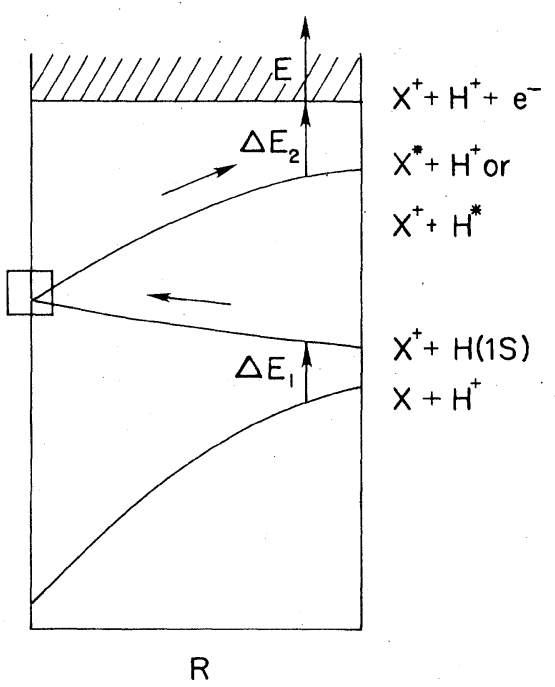

FIG. 14. Schematic correlation diagram showing proposed transitions accounting for exponential energy dependence of electron-ejection cross sections on electron energy (see text). 
While it is valid to choose $l_{1}$ and $l_{2}$ to be equal for outer-shell ionization, this is not correct for ejection of inner-shell electrons. For this case, we are dealing with the inner-shell diameter during transition 1 , but by the time transition 2 takes place the electron has been promoted to an outer shell. Thus $l_{2}$ should be the diameter of an outer shell, which leads to the use of the outer-shell binding energy in the exponential dependence on $E$. However, Figs. 10 and 11 indicate that the correct slope of the inner-shell ionization curves are obtained by using the corresponding inner-shell binding energies. This may be an indication that the second transition takes place instead near the united-atom limit, possibly in accord with a model developed by Briggs. ${ }^{23}$

It should also be noted that neither the empirical equation (2) nor the theoretical equation (6) yields $K$-shell cross sections that agree well with measured values. At low energies, experimental $K$ shell cross sections like those measured by Stolterfoht and Schneider ${ }^{24}$ follow an $E_{p}^{n}$ dependence with $n=2.5-3$, while the empirical equation yields $n=0.75$ or 1.0 and the theoretical equation gives a value of $n$ slightly greater than $\frac{5}{6}$. It appears that while Meyerhof's approach is useful for innershell ionization in heavy-ion collisions, for outershell ionization in proton collisions, and yields the correct distribution of electron energies in proton-induced inner-shell ionization, the dependence of the last process on proton energy is not correctly given.

\section{ACKNOWLEDGMENTS}

The author gratefully acknowledges many fruitful discussion with J. Macek which led to the application of molecular-orbital theory to this problem. Helpful discussions were also held with $\mathrm{K}$. Taulbjerg. C. A. Blocker, G. L. Webster, K. M. Bauer, B. L. Krauter, and E. P. Rudd assisted with the experimental work and the data processing. This work was supported by NSF Grants No. PHY76-23, 348 and PHY77-27, 523.
${ }^{1}$ See, for example, L. H. Toburen and W. E. Wilson, Phys. Rev. A 5, 247 (1972); L. H. Toburen, Steven T. Manson, and Yong-Ki Kim, ibid. 17, 148 (1978); M. E. Rudd, L. H. Toburen, and N. Stolterfoht, At. Data Nucl. Data Tables 18, 413 (1976) and the references contained therein.

${ }^{2}$ M. E. Rudd and D. H. Madison, Phys. Rev. A 14,128 (1976).

${ }^{3}$ T. L. Criswell, L. H. Toburen, and M. E. Rudd, Phys. Rev. A 16, 508 (1977).

${ }^{4}$ J. Macek, Phys. Rev. A 1, 235 (1970); G. B. Crooks and M. E. Rudd, Phys. Rev. Lett. 25, 1599 (1970).

${ }^{5} \mathrm{U}$. Fano and W. Lichten, Phys. Rev. Lett. 14, 627 (1965).

${ }^{6}$ J. B. Crooks and M. E. Rudd, Phys. Rev. A $\underline{3}, 1628$ (1971).

${ }^{7}$ L. H. Toburen, Phys. Rev. A 3 , 216 (1971).

${ }^{8}$ N. Stolterfoht, Z. Phys. $248, \overline{9} 2$ (1971).

${ }^{9}$ M. E. Rudd, C. A. Sautter, and C. L. Bailey, Phys. Rev. 151, 20 (1966).

${ }^{10}$ M. E. Rudd and T. Jorgensen, Jr., Phys. Rev. 131, 666 (1963).

${ }^{11} \mathrm{~K}$. Siegbahn, ESCA Applied to Free Molecules (E1sevier, Amsterdam, 1969).

${ }^{12}$ A. E. S. Green and R. J. McNeal, J. Geophys. Res. 76,
133 (1971).

${ }^{13}$ J. W. Hooper, E. W. McDaniel, D. W. Martin, and D. S. Harmer, Phys. Rev. 121, 1123 (1961); J. W. Hooper, D. S. Harmer, D. W. Martin, and E. W. McDaniel, ibid. 125, 2000 (1962); J. W. Hooper, thesis, Georgia Institute of Technology, 1961 (unpublished).

${ }^{14}$ F. J. DeHeer, J. Schutten, and H. Moustafa, Physica 32, 1766 (1966).

${ }^{15}$ E. S. Solov'ev, R. N. Il'in, V. A. Oparin, and N. V. Fedorenko, Sov. Phys. JETP 15, 459 (1962).

${ }^{16}$ H. B. Gilbody and A. R. Lee, Proc. R. Soc. A 274,365 (1963).

${ }^{17}$ V. V. Afrosimov, R. N. Il'in, and N. V. Fedorenko, Sov. Phys. JETP 34, 968 (1958).

${ }^{18}$ W. E. Meyerhof, Phys. Rev. Lett. 31, 1341 (1973).

${ }^{19}$ W. E. Meyerhof and Knud Taulbjerg, Ann. Rev. Nucl. Sci. 27, 279 (1975).

${ }^{20} \mathrm{~K}$. Taulbjerg, J. S. Briggs, and J. Vaaben, J. Phys. B 9, 1351 (1976).

${ }^{21}$ Yu. N. Demkov, Sov. Phys. JETP 18, 138 (1964).

${ }^{22} \mathrm{~J}$. Macek (private communication).

${ }^{23} \mathrm{~J}$. Briggs, J. Phys. B 8 , L485 (1975).

${ }^{24}$ N. Stolterfoht and D. Schneider, Phys. Rev. A 11, 721 (1975). 